\title{
Long-Term Outcome in Patients with a Solitary Peutz- Jeghers Polyp
}

\author{
Masaya Iwamuro ${ }^{D},{ }^{1}$ Yuki Aoyama, ${ }^{2}$ Seiyuu Suzuki, ${ }^{3}$ Sayo Kobayashi, ${ }^{4}$ \\ Tatsuya Toyokawa $\left(\mathbb{D},{ }^{5}\right.$ Yuki Moritou, ${ }^{6}$ Shinichiro Hori, ${ }^{7}$ Kazuhiro Matsueda, ${ }^{8}$ \\ Masao Yoshioka, ${ }^{9}$ Takehiro Tanaka, ${ }^{10}$ and Hiroyuki Okada ${ }^{1}$
}

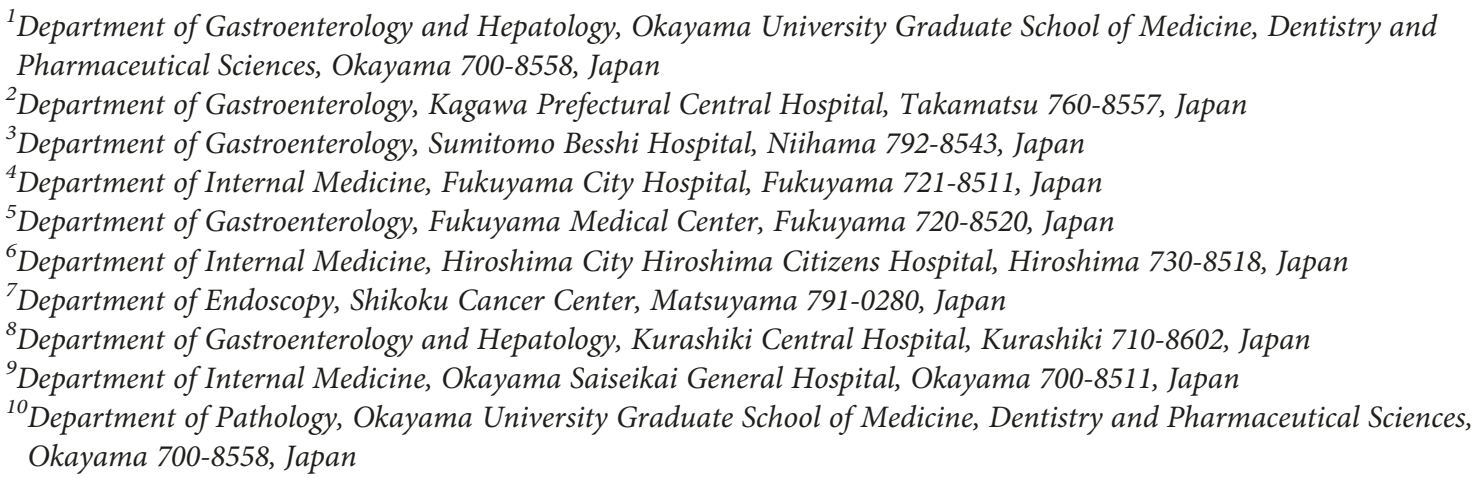

Correspondence should be addressed to Masaya Iwamuro; pr145h2k@okayama-u.ac.jp

Received 26 May 2019; Revised 7 August 2019; Accepted 29 August 2019; Published 8 September 2019

Academic Editor: Paolo Gionchetti

Copyright (c) 2019 Masaya Iwamuro et al. This is an open access article distributed under the Creative Commons Attribution License, which permits unrestricted use, distribution, and reproduction in any medium, provided the original work is properly cited.

\begin{abstract}
Background. Clinical characteristics and prognosis of patients with a solitary Peutz-Jeghers polyp (PJP) have not been fully investigated. Methods. Solitary PJP was diagnosed when a single hamartomatous lesion was identified in the gastrointestinal tract of patients without mucocutaneous pigmentation or a family history of Peutz-Jeghers syndrome. We retrospectively reviewed 51 patients ( 32 men and 19 women) with a solitary PJP and analyzed the sex, age at diagnosis, endoscopic features, and outcomes in this patient group. The STK11/LKB1 germline mutation was not investigated in any of the patients. Results. The mean age of the 51 patients was 66.1 years. The polyp was found in the duodenum $(N=10)$, jejunum $(N=2)$, cecum $(N=2)$, transverse colon $(N=5)$, sigmoid colon $(N=21)$, or rectum $(N=11)$. Most of the polyps presented as a pedunculated lesion $(N=40)$, followed by semipedunculated $(N=9)$ and sessile $(N=2)$ morphologies. The mean size of a solitary PJP was $15.6 \mathrm{~mm}$ (range: 5 to $33 \mathrm{~mm}$ ). During a mean endoscopic follow-up period of 4.5 years (range: 0.1 to 16.1 years), no recurrence was identified. Eighteen of the enrolled patients had a history of cancer or concomitant cancer. Five patients died due to nongastrointestinal-related causes. No additional cancer or death directly related to solitary PJP was observed. Conclusions. Solitary PJPs did not recur in this study. Although examination of the entire gastrointestinal tract using esophagogastroduodenoscopy, enteroscopy, and colonoscopy is desirable to exclude Peutz-Jeghers syndrome, follow-up endoscopy after endoscopic polyp resection may be unnecessary, once the diagnosis of a solitary PJP is made.
\end{abstract}

\section{Introduction}

Peutz-Jeghers syndrome is an autosomal dominant genetic disorder characterized by the development of multiple polyps in the gastrointestinal tract in association with patches of hyperpigmentation in the mouth and on the hands and feet $[1,2]$. The gastrointestinal polyps found in patients with Peutz-Jeghers syndrome are hamartomatous and 


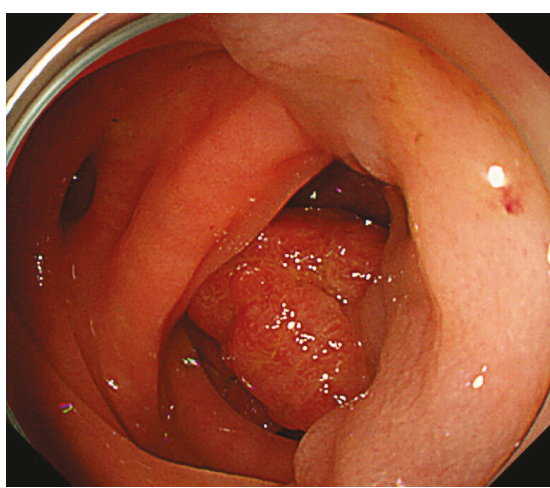

(a)

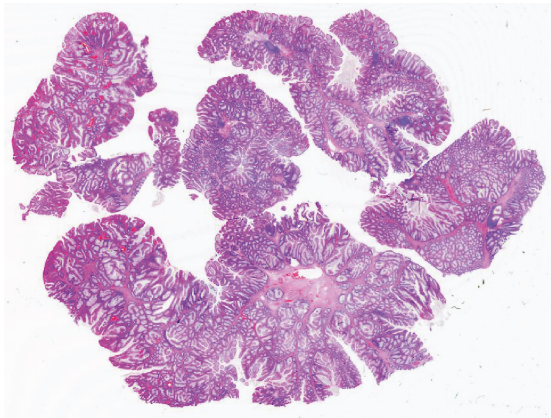

(c)

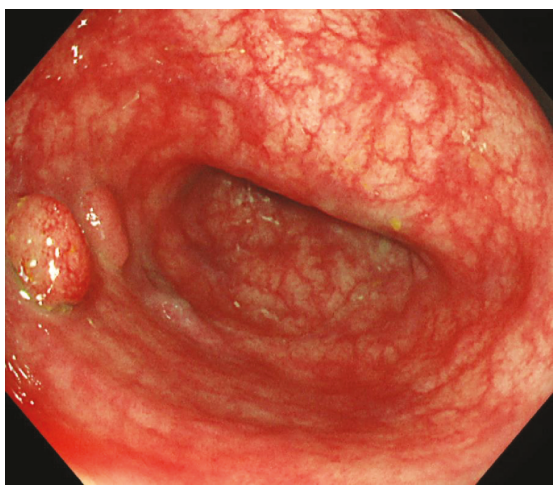

(e)

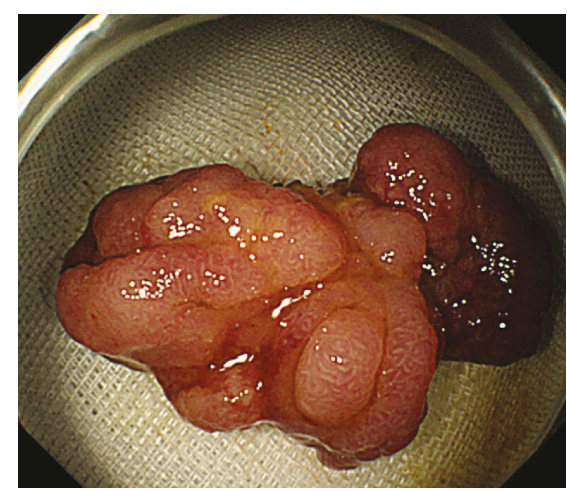

(b)

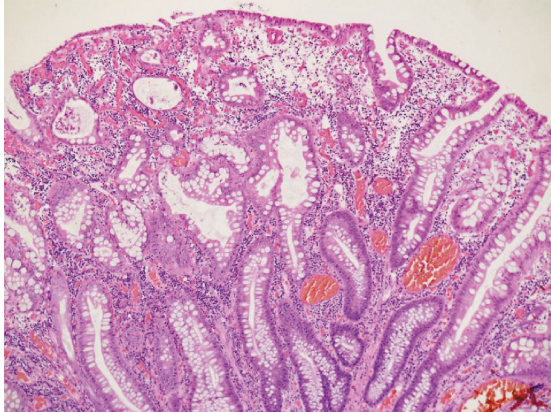

(d)

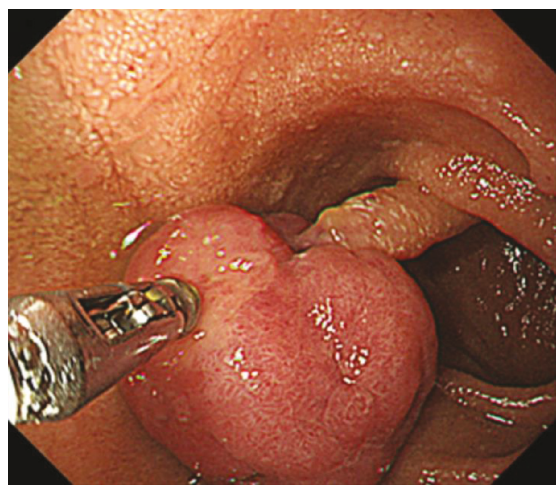

(f)

FIgURE 1: A solitary PJP in the sigmoid colon was endoscopically resected with a snare device (a, b). Pathological analysis revealed that the resected polyp was hamartomatous, showing arborization of smooth muscle within the lamina propria (c, d). A solitary PJP showing sessile morphology was observed in the sigmoid colon (e). In another patient, a pedunculated polyp in the duodenum was diagnosed as a solitary PJP (f).

are typically characterized by distinctive arborization of smooth muscle within the lamina propria [3]. These hamartomatous lesions can present as solitary polyps in the gastrointestinal tract in patients without mucocutaneous pigmentation and are termed solitary Peutz-Jeghers polyps (PJPs).

Owing to infrequency, the clinical characteristics and outcome of cases with a solitary PJP have not been fully investigated. In this report, we identified 51 patients with a solitary PJP from 9 institutions and summarized the endoscopic findings, presence or absence of malignant tumors in the follow-up period, and prognoses.

\section{Methods}

A solitary PJP was defined as a single hamartomatous lesion detected in the gastrointestinal tract of patients without a family history of Peutz-Jeghers syndrome or mucocutaneous pigmentation. Representative pathological features of PJP are extensive smooth-muscle proliferation and an elongated arborized pattern of polyp formation (Figure 1). Peutz-Jeghers syndrome was diagnosed when the patient had any of the following clinical criteria, and these patients were excluded from the study: 2 or more histologically confirmed PJPs, any number of PJPs detected 
in a person whose family history included 1 close relative with Peutz-Jeghers syndrome, characteristic mucocutaneous pigmentation in a person whose family history included 1 close relative with Peutz-Jeghers syndrome, and any number of PJPs in a person who also had characteristic mucocutaneous pigmentation [4-6].

We retrospectively reviewed 51 patients (32 men and 19 women) with a solitary PJP and analyzed the sex, age at diagnosis, endoscopic features, and outcomes in this patient group. Letters of inquiry regarding patients with solitary PJP were sent from the Department of Gastroenterology and Hepatology, Okayama University Graduate School of Medicine, Dentistry and Pharmaceutical Sciences, to 8 collaborating institutions. Patients with a pathologically diagnosed PJP in the gastrointestinal tract were included. Finally, a total of 51 patients diagnosed with a solitary PJP between January 1999 and April 2018 were identified and were retrospectively registered in this study. Three of the 51 patients examined also participated in our previous study [7]. Although the STK11/LKB1 germline mutation has been reported to be specific for Peutz-Jeghers syndrome, the presence or absence of the mutation was not investigated in any of the patients.

The follow-up period was defined as the time from diagnosis of PJP to death from any cause or the last hospital visit. The endoscopic follow-up period was defined as the time from resection of a solitary PJP to the last endoscopic examination to evaluate the intestinal segment with the PJP. Statistical analyses were performed using JMP 14.0.0 software (SAS Institute, Inc., Cary, NC, USA), and 1-way analysis of variance followed by a Tukey-Kramer post hoc test for multiple comparisons. $P<0.05$ was considered to indicate a statistically significant difference. The present study was approved by the Ethics Committee of Okayama University Hospital and adhered to the Declaration of Helsinki.

\section{Results}

Characteristics of the enrolled patients and the location, type, and size of the polyps are shown in Table 1 . The solitary PJP was found in the duodenum, jejunum, cecum, transverse colon, sigmoid colon, or rectum, while no cases of polyps in the esophagus, stomach, ileum, ascending colon, and descending colon were observed. Most of the polyps presented as a pedunculated lesion, followed by semipedunculated and sessile morphologies. Table 2 shows the distribution in the gastrointestinal tract and PJP morphology. With respect to polyp size in each intestinal segment, one-way analysis of variance followed by TukeyKramer post hoc testing found no differences in polyp sizes across locations.

Among those who underwent endoscopic examination, 48 patients (94\%) had colonoscopy and 33 (65\%) had esophagogastroduodenoscopy. Enteroscopy was performed in only 4 patients (8\%): 3 had video capsule enteroscopy alone and 1 patient with a PJP in the jejunum underwent peroral double-balloon enteroscopy and video capsule enteroscopy. In the latter patient, the jejunal polyp was diagnosed during video capsule enteroscopy and endoscopically resected dur-
TABLE 1: Clinical characteristics of study subjects.

\begin{tabular}{|c|c|}
\hline & $N$ \\
\hline \multicolumn{2}{|l|}{ Sex } \\
\hline Male & $32(63 \%)$ \\
\hline Female & $19(37 \%)$ \\
\hline Mean age (years) & 66.1 (range: $32-92$ ) \\
\hline \multicolumn{2}{|l|}{ Location } \\
\hline Esophagus & 0 \\
\hline Stomach & 0 \\
\hline Duodenum & $10(20 \%)$ \\
\hline Jejunum & $2(4 \%)$ \\
\hline Ileum & 0 \\
\hline Cecum & $2(4 \%)$ \\
\hline Ascending colon & 0 \\
\hline Transverse colon & $5(10 \%)$ \\
\hline Descending colon & 0 \\
\hline Sigmoid colon & $21(41 \%)$ \\
\hline Rectum & $11(22 \%)$ \\
\hline Mean size \pm SD $(\mathrm{mm})$ & $15.6 \pm 7.1$ (range: $3-33$ ) \\
\hline \multicolumn{2}{|l|}{ Morphology } \\
\hline Pedunculated & $40(78 \%)$ \\
\hline Semipedunculated & $9(18 \%)$ \\
\hline Sessile & $2(4 \%)$ \\
\hline \multicolumn{2}{|l|}{ Evaluation of recurrence } \\
\hline Done & $25(49 \%)$ \\
\hline Not done & $26(51 \%)$ \\
\hline Recurrence of PJ polyps & 0 \\
\hline $\begin{array}{l}\text { Mean endoscopic follow-up } \\
\text { period (years) }\end{array}$ & 3.1 (range: $0.1-16.1$ ) \\
\hline \multicolumn{2}{|l|}{ Outcome } \\
\hline Alive & $46(90 \%)$ \\
\hline Died of other cause* & $5(10 \%)$ \\
\hline Mean follow-up period (years) & 3.8 (range: $0.0-18.8$ ) \\
\hline
\end{tabular}

${ }^{*}$ Hypopharyngeal cancer, interstitial pneumonia, pulmonary thrombosis, sepsis, and unknown cause.

ing a double-balloon enteroscopy. The other patient with a PJP in the jejunum underwent colectomy for ascending colon cancer, and the polyp was diagnosed during a laparotomy via digital examination of the small intestine. Segmental resection of the jejunum was performed to remove the polyp. Intraoperative endoscopy revealed no other polyps in the small intestine.

Twenty-one malignancies were diagnosed in 18 patients (35\%) before the solitary PJP was identified. Sixteen of the malignancies were cancers also seen in patients with PeutzJeghers syndrome: 12 cancers of the gastrointestinal tract, including gastric cancer $(N=5)$, cecal cancer $(N=1)$, colon cancer $(N=4)$, and rectal cancer $(N=2)$. Breast cancer was found in two patients whereas lung and thyroid cancers were seen in one case each. In addition, prostate cancer $(N=3)$, hypopharyngeal cancer $(N=1)$, and liver cancer $(N=1)$ were found in the enrolled patients. These had been diagnosed prior to the identification of PJP in all patients, and 
TABLE 2: Gastrointestinal tract involvement and PJP morphology.

\begin{tabular}{lccccccc}
\hline & Duodenum & Jejunum & Cecum & Transverse colon & Sigmoid colon & Rectum & Total \\
\hline Ip & 7 & 2 & 1 & 5 & 18 & 7 & 40 \\
Isp & 3 & 0 & 0 & 0 & 0 & 1 & 4 \\
Is & 0 & 0 & 1 & 5 & 21 & 0 \\
Total & 10 & 2 & 2 & 51 & 11 \\
\hline
\end{tabular}

Ip: pedunculated; Isp: semipedunculated; Is: sessile.

no cancers were diagnosed during the follow-up after resection of the solitary PJP.

The solitary PJP was endoscopically resected and endoscopically followed up in 25 patients (49\%). Follow-up endoscopy was not performed in the other 26 patients. During a mean endoscopic follow-up of 4.5 years (range: 0.1 to 16.1 years), no recurrence was identified. Patients were followed up for an average of 3.8 years (range: 0.0 to 18.8 years) to determine prognosis. Five patients died as follows: hypopharyngeal cancer $(N=1)$, interstitial pneumonia $(N=1)$, pulmonary thrombosis $(N=1)$, sepsis $(N=1)$, or unknown cause $(N=1)$. All other patients were alive as of the last scheduled follow-up.

\section{Discussion}

To our knowledge, this is the largest study to date on patients with a solitary PJP. In the 51 patients in this study, the sigmoid colon was most frequently involved, followed by the rectum, duodenum, transverse colon, jejunum, and cecum. Overall, a solitary PJP was found in the large intestine in more than three-quarters of the patients, e.g., cecum, transverse colon, sigmoid colon, and rectum $(N=39,77 \%)$. Although gastric and ileal involvement was not observed in this study, cases with a solitary PJP in the stomach [8] and ileum [9] have been reported. However, to our knowledge, no patients have had a solitary PJP in the esophagus. Patients with Peutz-Jeghers syndrome typically have multiple hamartomatous polyps in the stomach, small intestine, and/or large intestine [2]. Thus, as in Peutz-Jeghers syndrome, the esophagus does not seem to be affected by solitary PJPs.

Although 5 patients died of causes unrelated to PJP, the remaining 46 were alive as of the last scheduled follow-up. In addition, no recurrence was detected on follow-up endoscopy in 25 patients. Oncel et al. reviewed 8 patients with a solitary PJP and found no recurrences during a median of 11.5 years (range: 3 to 22 years) [10]. They also noted that 3 patients died of causes other than PJP, while the other 5 were alive. These results suggest that a solitary PJP does not recur. When a single polyp showing pathological features consistent with PJP is identified in the gastrointestinal tract, physical examination to check for mucocutaneous pigmentation and a review of family history are essential. In addition, we considered that examination of the entire gastrointestinal tract using esophagogastroduodenoscopy, enteroscopy, and colonoscopy is desirable to exclude Peutz-Jeghers syndrome. Once the diagnosis of a solitary PJP is made, follow-up endoscopy after endoscopic resection of the polyp may be unnecessary.
Patients with Peutz-Jeghers syndrome reportedly have a lifetime cumulative risk of up to $93 \%$ for development of malignancies, such as colorectal, breast, small bowel, gastric, and pancreatic cancers [2, 11, 12]. Ishida et al. reviewed 583 Japanese patients with Peutz-Jeghers syndrome reported in the literature and estimated the cumulative risk of a malignant tumor as $83.0 \%$ at 70 years of age [6]. In the present study, the polyps were often detected in patients with cancer: patients had gastrointestinal, prostate, breast, lung, thyroid, hypopharyngeal, and liver cancers. However, these had been diagnosed prior to identification of a PJP in all enrolled patients. Although no additional cancer or mortality directly related to solitary PJPs was observed in this study, the follow-up time was rather short in some cases and only half of the patients underwent a follow-up endoscopy. Therefore, further investigation is required to determine the relationship between solitary PJPs and malignancies.

This study had several limitations. First, not all patients had endoscopic evaluation of the entire gastrointestinal tract. In particular, the small intestine was not evaluated in 47 patients, although polyposis in the small bowel is frequently observed in Peutz-Jeghers syndrome. Second, the presence of STK11/LKB1 germline mutations, which are specific for Peutz-Jeghers syndrome, was not investigated in solitary PJP patients. In Japan, unfortunately, genetic testing is virtually unavailable in clinical settings, because it is not covered by health insurance. As a result, several patients may have had undiagnosed multiple PJPs or STK11/LKB1 germline mutations; thus, true Peutz-Jeghers syndrome may have been misdiagnosed as solitary PJP. Third, malignant diseases had been diagnosed in a subset of the enrolled patients prior to the detection of PJP. Fourth, follow-up endoscopy was performed in 25 patients, while endoscopy examinations were not repeated in the remaining 26 patients. In addition, the observation period of 3.8 years is relatively short. A complete follow-up with a longer observation period is desirable to accurately estimate the recurrence rate, prognosis, and the frequency of malignant tumors.

\section{Conclusions}

We retrospectively investigated 51 patients with a solitary PJP. Although 5 patients died of causes unrelated to a solitary PJP, the other patients were alive as of the last scheduled follow-up. During a mean endoscopic followup of 4.5 years, no recurrence was observed, indicating that solitary PJPs do not recur. 


\section{Data Availability}

The clinical data used to support the findings of this study are available from the corresponding author upon request.

\section{Conflicts of Interest}

No potential conflict of interest relevant to this article was reported.

\section{Authors' Contributions}

MI worked on conception and design, data collection, analysis, interpretation of the data, drafting of the article, critical revision of the article for important intellectual content, and final approval. YA, SS, SK, T Toyokawa, YM, SH, KM, and MY worked on data collection and final approval. T Tanaka worked on data collection, critical revision of the article for important intellectual content, and final approval. $\mathrm{HO}$ worked on critical revision of the article for important intellectual content and final approval.

\section{References}

[1] J. Daniell, J. P. Plazzer, A. Perera, and F. Macrae, "An exploration of genotype-phenotype link between Peutz-Jeghers syndrome and STK11: a review," Familial Cancer, vol. 17, no. 3, pp. 421-427, 2018.

[2] S. X. Duan, G. H. Wang, J. Zhong et al., "Peutz-Jeghers syndrome with intermittent upper intestinal obstruction: a case report and review of the literature," Medicine, vol. 96, no. 17, article e6538, 2017.

[3] J. Y. Tse, S. Wu, S. A. Shinagare et al., "Peutz-Jeghers syndrome: a critical look at colonic Peutz-Jeghers polyps," Modern Pathology, vol. 26, no. 9, pp. 1235-1240, 2013.

[4] L. A. Aaltonen, "Hereditary intestinal cancer," Seminars in Cancer Biology, vol. 10, no. 4, pp. 289-298, 2000.

[5] S. Aretz, D. Stienen, S. Uhlhaas et al., "High proportion of large genomic STK11 deletions in Peutz-Jeghers syndrome," Human Mutation, vol. 26, no. 6, pp. 513-519, 2005.

[6] H. Ishida, Y. Tajima, T. Gonda, K. Kumamoto, K. Ishibashi, and T. Iwama, "Update on our investigation of malignant tumors associated with Peutz-Jeghers syndrome in Japan," Surgery Today, vol. 46, no. 11, pp. 1231-1242, 2016.

[7] S. Suzuki, S. Hirasaki, F. Ikeda, E. Yumoto, H. Yamane, and M. Matsubara, "Three cases of solitary Peutz-Jeghers-type hamartomatous polyp in the duodenum," World Journal of Gastroenterology, vol. 14, no. 06, pp. 944-947, 2008.

[8] L. Harbaum, J. B. Geigl, H. Volkholz et al., "Sporadic gastric Peutz-Jeghers polyp with intraepithelial neoplasia," APMIS, vol. 117, no. 12, pp. 941-943, 2009.

[9] A. P. Srinivasan, B. O. Parijatham, and H. Ganapathy, "A case of intestinal MALToma with co-existent tuberculosis and Peutz-Jeghers polyp," Journal of Postgraduate Medicine, vol. 61, no. 2, pp. 134-136, 2015.

[10] M. Oncel, F. H. Remzi, J. M. Church, J. Goldblum, M. Zutshi, and V. Fazio, "Course and follow-up of solitary Peutz-Jeghers polyps: a case series," International Journal of Colorectal Disease, vol. 18, no. 1, pp. 33-35, 2003.
[11] R. Y. Fan and J. Q. Sheng, "A case of Peutz-Jeghers syndrome associated with high-grade intramucosal neoplasia," International Journal of Clinical and Experimental Pathology, vol. 8, no. 6, pp. 7503-7505, 2015.

[12] M. G. F. van Lier, A. Wagner, E. M. H. Mathus-Vliegen, E. J. Kuipers, E. W. Steyerberg, and M. E. van Leerdam, "High cancer risk in Peutz-Jeghers syndrome: a systematic review and surveillance recommendations," American Journal of Gastroenterology, vol. 105, no. 6, pp. 1258-1264, 2010. 


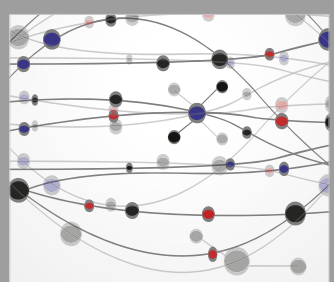

The Scientific World Journal
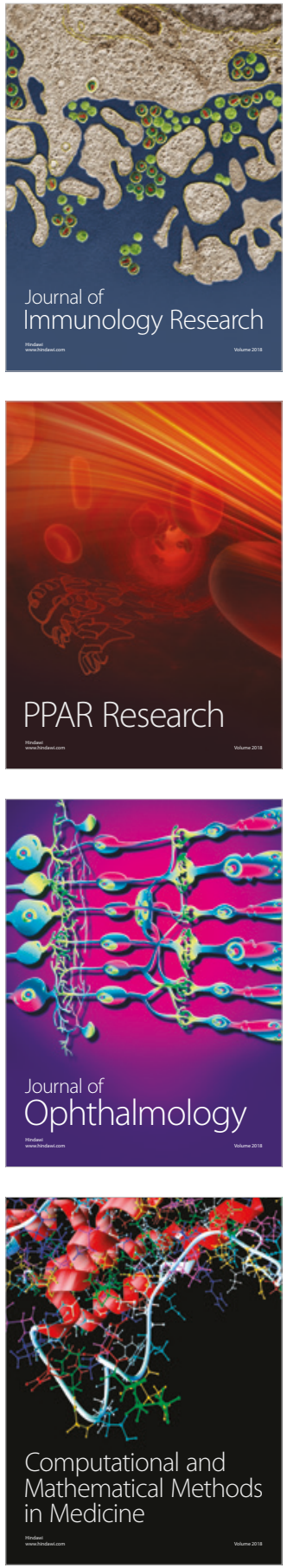

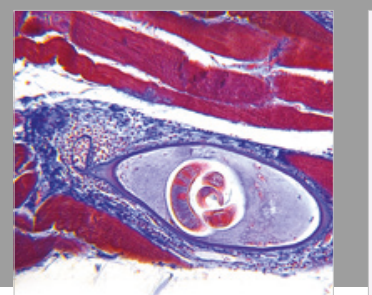

Gastroenterology Research and Practice

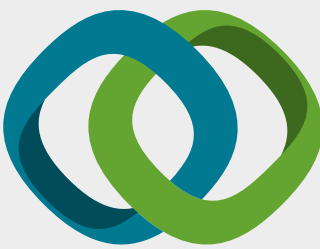

\section{Hindawi}

Submit your manuscripts at

www.hindawi.com
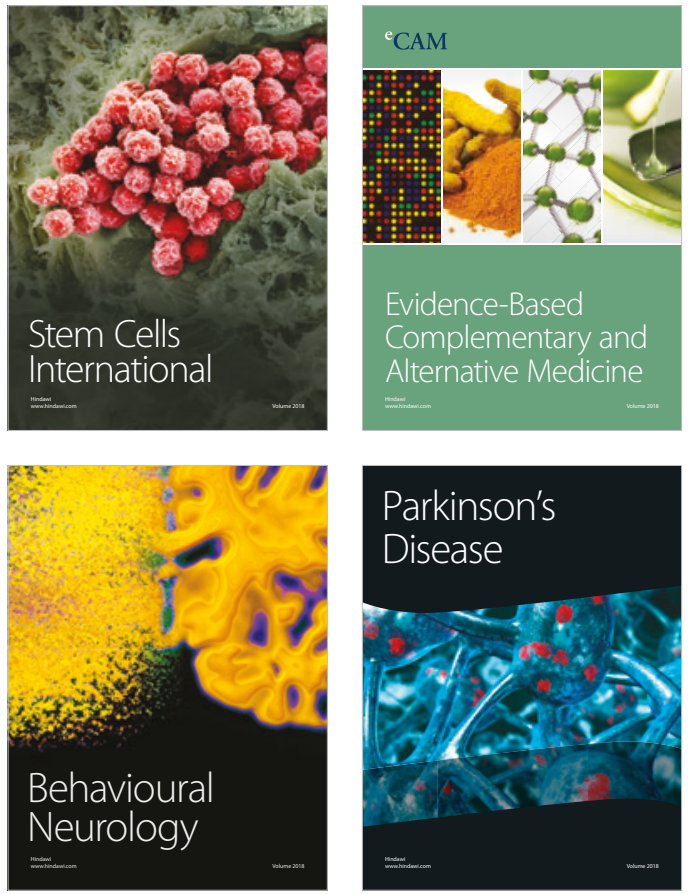

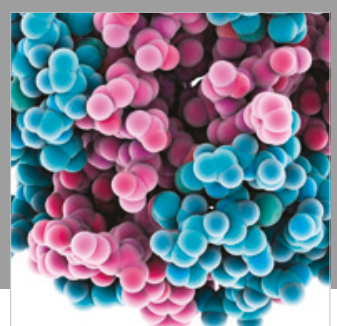

ournal of

Diabetes Research

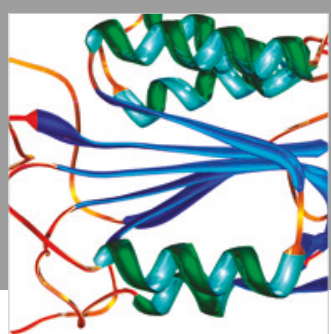

Disease Markers
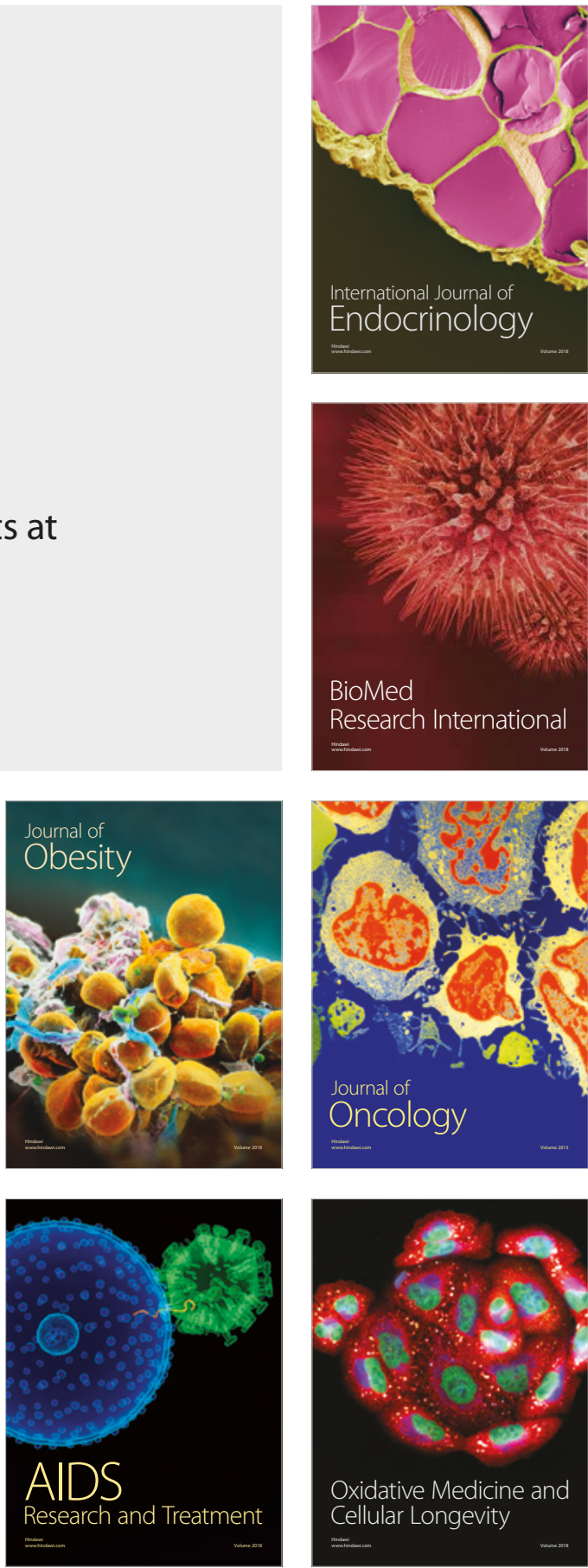